\title{
A micro-computed tomographic evaluation of dentinal microcrack alterations during root canal preparation using single-file Ni-Ti systems
}

\author{
MEI-LIN LI ${ }^{1}$, WEI-LI LIAO ${ }^{2}$ and HUA-XIONG CAI ${ }^{2}$ \\ ${ }^{1}$ Department of Oral Medicine, Haizhu Square Branch, Stomatological Hospital, Southern Medical University \\ (Guangdong Provincial Stomatological Hospital), Guangzhou, Guangdong 510280; \\ ${ }^{2}$ Zhujiang New Town Dental Clinic, Guanghua School of Stomatology, Guangdong Provincial Key Laboratory \\ of Stomatology, Sun Yat-Sen University, Guangzhou, Guangdong 510060, P.R. China
}

Received January 20, 2017; Accepted October 26, 2017

DOI: 10.3892/etm.2017.5399

\begin{abstract}
The aim of the present study was to evaluate the length of dentinal microcracks observed prior to and following root canal preparation with different single-file nickel-titanium (Ni-Ti) systems using micro-computed tomography (micro-CT) analysis. A total of 80 mesial roots of mandibular first molars presenting with type II Vertucci canal configurations were scanned at an isotropic resolution of $7.4 \mu \mathrm{m}$. The samples were randomly assigned into four groups ( $n=20$ per group) according to the system used for root canal preparation, including the WaveOne (WO), OneShape (OS), Reciproc (RE) and control groups. A second micro-CT scan was conducted after the root canals were prepared with size 25 instruments. Pre- and postoperative cross-section images of the roots $(n=237,760)$ were then screened to identify the lengths of the microcracks. The results indicated that the microcrack lengths were notably increased following root canal preparation $(\mathrm{P}<0.05)$. The alterations in microcrack length in the OS group were more significant compared with those in the WO, RE and control groups $(\mathrm{P}<0.05)$. In conclusion, the formation and development of dentinal microcracks may be associated with the movement caused by preparation rather than the taper of the files. Among the single-file Ni-Ti systems, WO and RE were not observed to cause notable microcracks, while the OS system resulted in evident microcracks.
\end{abstract}

Correspondence to: Professor Hua-Xiong Cai, Zhujiang New Town Dental Clinic, Guanghua School of Stomatology, Guangdong Provincial Key Laboratory of Stomatology, Sun Yat-Sen University, 56 Lingyuanxi Road, Guangzhou, Guangdong 510060, P.R. China E-mail: caihx@mail.sysu.edu.cn

Key words: single-file nickel-titanium, root canal preparation, dentinal microcrack, micro-computed tomography

\section{Introduction}

The major purpose of root canal therapy is to reduce the intracanal microorganisms. Chemo-mechanical preparation is an essential and indispensable step in disinfecting the root canal system (1). During endodontic treatment, the roots are susceptible to dentinal damage. Various factors, including the physical properties of the teeth, the endodontic instruments and the preparation technique used, contribute to this damage (2).

In addition to stainless steel hand files, several rotary nickel-titanium (Ni-Ti) file systems have been introduced for the preparation of root canals (3). Ni-Ti instruments have numerous advantages over conventional files, including increased flexibility and a shorter working time (3). However, these systems have different tip designs, tapers and cutting blade configurations that place stress on the root canal walls and may lead to microcracks or craze lines, which may develop into fractures due to repeated stress from occlusal forces (4) and may then lead to tooth loss. Three recently introduced single-file Ni-Ti systems, including WaveOne (WO), OneShape (OS) and Reciproc (RE), enable canal preparation using only one instrument and require less time in comparison with full-sequence rotary instrument systems. However, Kishen (5) reported that cracks may also form in untreated teeth due to the patient age (6), gender, masticatory function or occlusal trauma. Certain studies have indicated that root fracture is connected with dentinal removal $(3,7,8)$, whereas other researchers have not supported this theory $(9,10)$. The movement caused by the preparation method, the design and taper of the file, and the preparation time lead to different degrees of microcracks $(9,11-13)$.

The primary techniques currently used to observe dentinal microcracks are stereoscopic microscopy (14), scanning electron microscopy (SEM), staining, infrared imaging and micro-computed tomography (micro-CT). SEM is typically used to observe the slice of a root $(11,15,16)$; however, cracks may be formed at the root during both sample preparation and the period of observation. Since microcracks can extend through every slice or remain on the surface, SEM may miss microcracks in the slices, which limits its use in dentinal 
microcrack observation. Furthermore, stereoscopic microscopy, staining and infrared imaging do not reveal cracks with a micro-scale resolution $(17,18)$.

Micro-CT is a multi-functional three-dimensional scanning method that offers high resolution; thus, the use of micro-CT in dental analyses is increasing. In recent years, micro-CT has enabled novel possibilities for endodontic research by allowing nondestructive volumetric quantitative and qualitative assessments prior to and following different endodontic procedures $(9,19)$.

The present study evaluated the alterations observed in dentinal microcracks following root canal preparation with three different single-file Ni-Ti file systems using micro-CT analysis. A hand k-file system was used as a reference technique for comparison.

\section{Materials and methods}

Selection of the specimens. A total of 100 human mandibular first molars with completely separated roots, which were extracted for reasons unrelated to the present study, were obtained from a pool of teeth between April 2016 and June 2016 from a total of 92 patients at the Guanghua School and Hospital of Stomatology (Guangzhou, China). Teeth were stored in $0.9 \%$ normal saline at $5^{\circ} \mathrm{C}$. All patients (aged 20-70 years old; 52 male:40 female) provided informed consents, and the experiments were approved by the local ethics committee of Guanghua School and Hospital of Stomatology, Sun Yat sen University.

For the selection of samples, the roots were initially inspected by stereomicroscopy under a magnification of x 12 to exclude teeth with pre-existing craze lines or cracks. A digital radiography scan in a buccolingual direction was performed to determine the curvature angle of the mesial root using an open-source image analysis program (Fiji version $1.47 \mathrm{n}$ software; Fiji, Madison, WI, USA). Only teeth with a moderate curvature of the mesial root (ranging between $10^{\circ}$ and $20^{\circ}$ ) were selected. Teeth without patency for the canal length, as determined by a size $10 \mathrm{k}$-file (Dentsply Maillefer, Tulsa, OK, USA), were also discarded. The coronal portions and distal roots of all teeth were removed using a low-speed saw (IsoMet; Buehler, Lake Bluff, IL, USA) with water cooling. Mesial roots of $\sim 11 \pm 1 \mathrm{~mm}$ in length were left to prevent the introduction of confounding variables. As a result, 100 specimens were selected and stored in $0.9 \%$ normal saline at $5^{\circ} \mathrm{C}$.

Micro-CT scanning. In order to obtain an overall outline of the anatomic configuration of the mesial canals, specimens were pre-scanned at a relatively low isotropic resolution (70 mm) using a micro-CT scanner ( $\mu$ CT 50; Scanco Medical, Brüttisellen, Switzerland) at $70 \mathrm{kV}$ and $114 \mathrm{~mA}$. Based on this pre-scan set of images, 80 specimens with type II Vertucci canal configurations were selected. These specimens were scanned again at an isotropic resolution of $7.4 \mathrm{~mm}$. Flat-field correction was performed prior to the scanning procedure in order to correct for variations in the camera pixel sensitivity. Scanning was performed by $360^{\circ}$ rotation around the vertical axis with a rotation step of $0.5^{\circ}$. The X-ray source was an air-cooled, sealed, microfocus X-ray tube with a focal spot size of $5 \mu \mathrm{m}$. X-rays were filtered with a $0.5-\mathrm{mm}$ aluminum filter, and the X-ray tube was operated at $70 \mathrm{kV}$ and $228 \mu \mathrm{A}$. The X-ray detector comprised a 2,048x2,048 pixel, 16-bit charge-coupled device camera with fiber-optic coupling to an X-ray scintillator. The system, which was controlled with a PC workstation running the Microsoft Windows XP Professional operating system (Microsoft Corp., Redmond, WA, USA), was used to acquire 1,300-1,600 transverse cross-sections per tooth in a bitmap format.

Root canal preparation. A thin film of polyether impression material was used to coat the cement surface of the roots to simulate the periodontal ligament. Each specimen was placed coronal-apically inside a custom-made epoxy resin holder (diameter, $18 \mathrm{~mm}$ ) to further streamline the co-registration processes. Apical patency was determined by inserting a size $10 \mathrm{k}$-file (size 10, 0.02 taper) $(9,15)$ into the root canal until its tip was visible at the apical foramen, then the length of the file was measured from the apical foramen to the cross section, and the working length (WL) was $0.5 \mathrm{~mm}$ shorter than the length of the file. Subsequent to establishing glidepaths with a length up to the WL using a size $15 \mathrm{k}$-file (size 15, 0.02 taper) (Dentsply Maillefer), the specimens were randomly assigned to three experimental groups and a control group ( $n=20$ per group) according to the system used for root canal preparation. The groups were as follows: WO group, in which the WO Ni-Ti reciprocating instrument (Dentsply Maillefer, Tulsa, OK, USA) was used; OS group, in which the OS Ni-Ti rotary instrument (Micro-Mega, Besançon, France) was used; RE group, in which the Reciproc Ni-Ti reciprocating instrument (VDW GmbH, Munich, Germany) was used; and the control group, in which a stainless steel root canal file (Dentsply Maillefer) was used.

In all groups, irrigation was performed using $40 \mathrm{ml}$ $(5.25 \%)$ sodium hypochlorite. Instruments were driven with the X-Smart plus motor (Dentsply Maillefer) according to each manufacturer's protocol, and a single experienced operator performed all the preparations. The apical sizes and tapers of the Ni-Ti preparation systems are shown in Table I.

In the WO group, the WO instrument (size 25, 0.08 taper) was moved in the apical direction using a slow in- and -out pecking motion of $\sim 3 \mathrm{~mm}$ in amplitude with light apical pressure in a reciprocating motion until the WL was reached. The instrument was then removed from the canal and cleaned. The specimens in the OS group were prepared with the OS instrument (size 25, 0.06 taper) using rotary motion to reach $2 / 3$ of the WL value, the WL-3 mm and the WL. In the RE group, the Reciproc instrument (size 25, 0.08 taper) was moved as described for the WO group. The control group was also prepared in a standard manner with a stainless steel root canal file until the WL was reached. The following sequence was used: A size $20 \mathrm{k}$-file (size 20, 0.02 taper) and a size $25 \mathrm{k}$-file (size 25, 0.02 taper). Subsequent to four steady strokes, the instrument was removed from the canal. Next, 17\% EDTA was used to wipe off the smear layer, and $0.9 \%$ normal saline was used to finish the preparation. Micro-CT scans of all samples were then performed using the aforementioned parameters.

Dentinal microcrack measurement. The cross-section images of the mesial roots from the furcation level to the apex $(n=297,200)$ were observed with ImageJ image processing 
Table I. Apical sizes and tapers of different nickel-titanium preparation systems.

\begin{tabular}{lcc}
\hline Preparation system & Apical size (mm) & Taper (\%) \\
\hline WaveOne & 0.25 & 8 \\
OneShape & 0.25 & 6 \\
RE & 0.25 & 8 \\
\hline
\end{tabular}

Table II. Length of microcracks in all groups.

\begin{tabular}{lccc}
\hline & \multicolumn{2}{c}{ Microcrack length } & \\
\cline { 2 - 3 } Group & $\begin{array}{c}\text { No preparation, } \\
\mu \mathrm{m}\end{array}$ & $\begin{array}{c}\text { Prepared to } \\
\text { size 25, } \mu \mathrm{m}\end{array}$ & P-value \\
\hline WO & $576.097 \pm 233.310$ & $1745.492 \pm 293.933$ & 0.12 \\
OS & $456.928 \pm 200.030$ & $2763.932 \pm 333.685^{\mathrm{a}}$ & 0.011 \\
RE & $626.044 \pm 259.122$ & $1822.519 \pm 370.132$ & 0.078 \\
Control & $657.710 \pm 202.638$ & $1521.711 \pm 392.589$ & 0.054
\end{tabular}

${ }^{\mathrm{a}} \mathrm{P}<0.05$ vs. no preparation. WO, WaveOne; OS, OneShape; RE, Reciproc.

software (National Institutes of Health, Bethesda, MD, USA) in order to analyze the type and distribution of microcracks. The images were screened by three ImageJ trained examiners to measure the dentinal microcrack lengths according to the length of the black line in the slice that was measured, as demonstrated in Fig. 1. To validate the screening process, image analyses were repeated twice at 2 -week intervals. In cases of disagreement among the examiners, the images were re-examined until agreement was reached. The samples were divided evenly into the coronal, medial and apical parts. The percentage, which was determined by the microcrack length of one part divided by the length of the entire sample, was quantified as the distribution of microcracks.

Statistical analysis. The data were statistically analyzed using SPSS software (version 19.0 for Windows; IBM Corp., Armonk, NY, USA). All data were presented normal distribution and homogeneity of variance. The lengths of preoperative dentinal microcracks were analyzed using analysis of variance. Alterations in microcrack lengths prior to and following preparation within the same group were analyzed using a paired t-test. Differences in the dentin microcrack lengths were assessed using a Student-Newman-Keuls (SNK) test. P $<0.05$ indicated that the differences were statistically significant.

\section{Results}

Microcrack classification. ImageJ processing software was used to observe the morphology of each sample. As shown in Fig. 2, the microcracks were classified as follows: Complete, originating from the root canal and extending to the root wall (black arrow); incomplete, originating from the root canal and

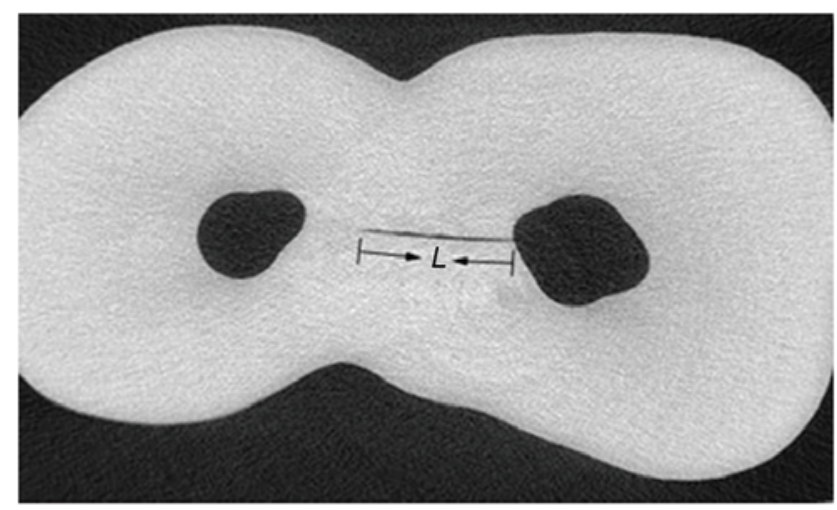

Figure 1. Micro-computed tomography image used for the measurement of the microcrack length, indicated by L.

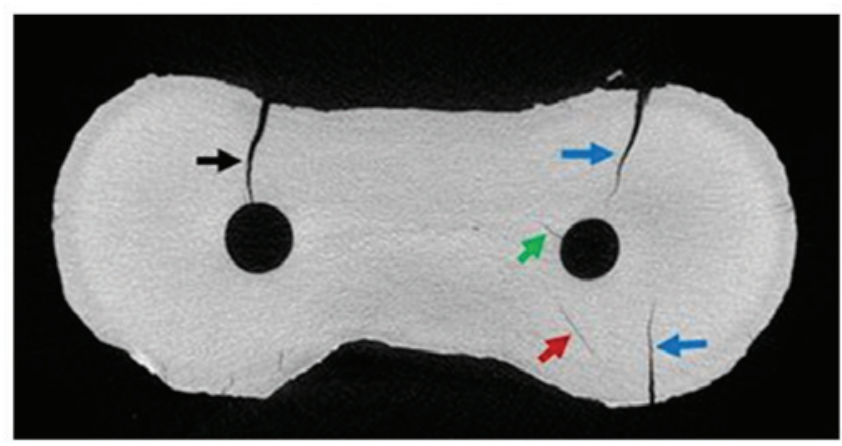

Figure 2. Micro-computed tomography image used to examine the morphology of the dentinal microcracks. Complete microcrack, originating from the root canal and extending to the root wall (black arrow); incomplete microcrack, originating from the root canal and not extending to the root wall (green arrow); or in-dentine microcrack, indicating microcracks present only in the dentine or originating from the root wall without reaching the root canal (red and blue arrows, respectively).

not extending to the root wall (green arrow); or in-dentine, indicating microcracks present only in the dentine or originating from the root wall without reaching the root canal (red and blue arrows, respectively) (20).

Microcrack length. The lengths of the dentin microcracks without preparation were not statistically significant $(\mathrm{P}>0.05)$. A paired $t$ test was used to analyze changes in the microcrack length prior to and following preparation. When compared with the length prior to preparation, the OS group length was significantly increased after preparation $(\mathrm{P}<0.05)$, whereas there were no significant changes in the WO, RE and control groups ( $\mathrm{P}>0.05$; Table II). Furthermore, as determined by the SNK test, the differences in the dentin microcrack lengths when prepared to size 25 between the three groups (WO, RE and control) and the OS group were statistically significant $(\mathrm{P}<0.05$; Table II). Similarly, Figs. 3 and 4 also indicated the lengths of microcracks were markedly increased in the OS after preparation compared with the other groups (Fig 3).

Distribution of microcracks. Samples were trisected, the length of each section was collected and the distribution of microcracks as a percentage of the total length was expressed. As illustrated in Table III, the distribution changes of 


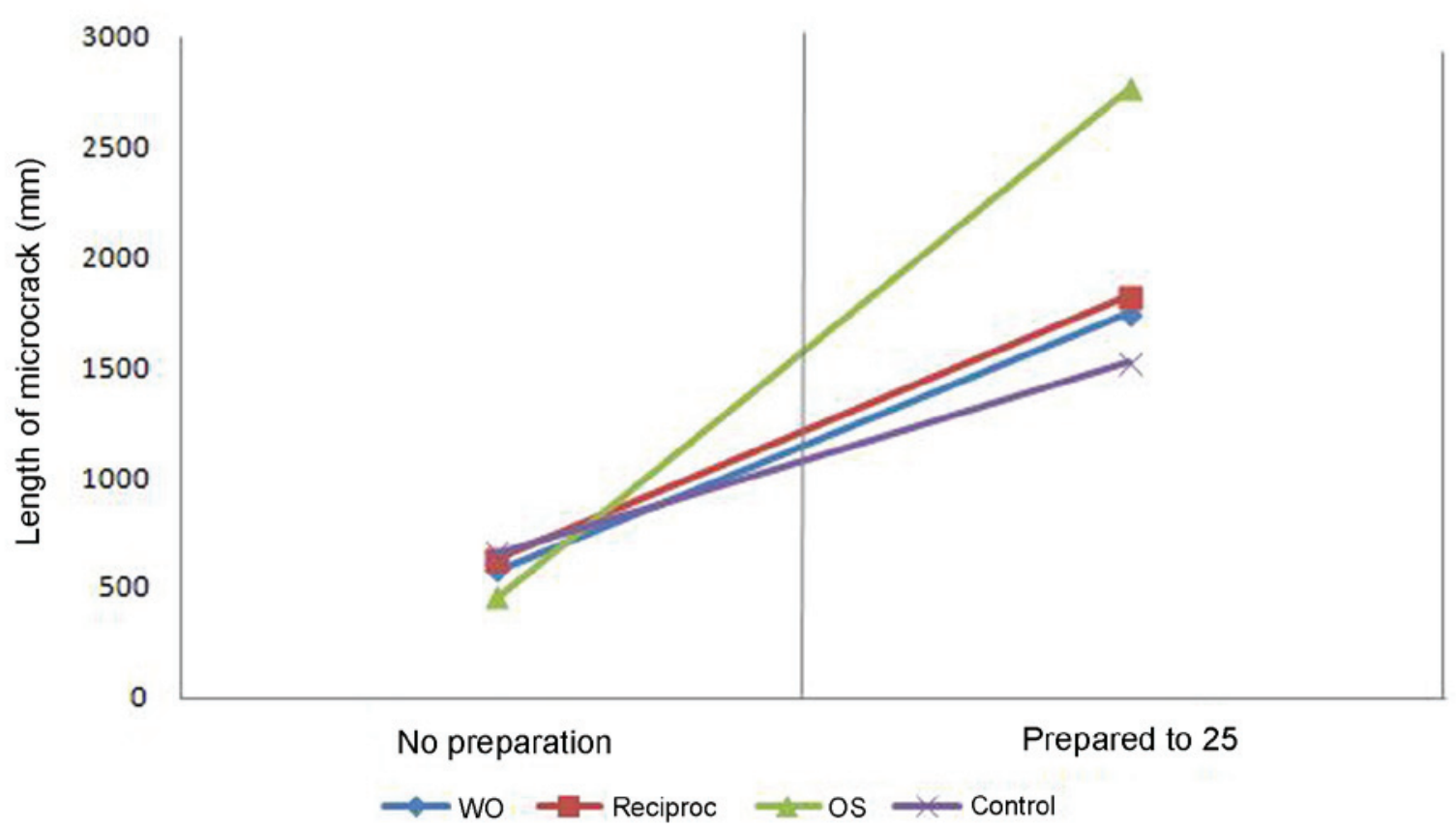

Figure 3. Length of microcracks prior to and post-preparation.

microcracks in the OS group was primarily observed in the apical and coronal parts of the samples. Others groups exhibited no distribution changes in the apical, coronal or medial parts.

\section{Discussion}

A novel technique that uses reciprocating motion has been previously proposed for root canal preparation (21). This approach relieves the stress on the instrument through counterclockwise and clockwise movements and, therefore, increases its resistance to cyclic fatigue compared with the traditional continuous rotation motion $(22,23)$. The WO and RE instruments, which were designed by different manufactures, are the main examples of commercially available single-file reciprocating Ni-Ti systems for root canal preparation that alternate between different values of counterclockwise and clockwise rotation movements, which allows for $360^{\circ}$ preparation subsequent to a series of reciprocating movements $(24,25)$. In addition, the OS instrument was designed using a single file and a rotary movement to complete preparation.

Previous studies have demonstrated a high rate of dentinal defects caused by the mechanical preparation of root canals $(15,26)$. Bürklein et al (12) demonstrated that root canal preparation with both rotary and reciprocating instruments resulted in dentinal defects. In addition, at the apical level, reciprocating files produced significantly more incomplete dentinal cracks as compared with those produced by full-sequence rotary systems. By contrast, Liu et al (13) used a similar methodology and observed that the ProTaper multiple-file rotary system caused an increased number of cracks on the apical root surface and in the canal wall in comparison with single-file rotary or reciprocating systems. Ashwinkumar et al (15) also observed that canal preparation with ProTaper rotary files was associated with significantly more microcracks compared with the WO reciprocating system.

Studies correlating mechanical preparation and the development of dentinal defects are based only on root-sectioning methods and direct observation by optical microscopy $(11,15,16)$. These methods undoubtedly have significant limitations associated with the destructive nature of the experiment, as reported in previous studies $(11-13,15,16)$. In previous results in which unprepared teeth were used, their control groups appeared to be validated as effective control groups; however, as no dentinal defects were detected, this type of control does not consider the potential damage produced by the interplay among the four sources of stress on the root dentin, including mechanical preparation, a chemical attack with sodium hypochlorite-based irrigation, sectioning procedures and dehydrogenation drying procedures (27).

In the present study, micro-CT imaging technology was used to evaluate the length of dentinal defects at the baseline and to compare the thickness of the dentine. This highly accurate and non-destructive method enables the assessment of specimens prior to preparation. Therefore, pre-existing cracks can be detected, and it is possible to determine the precise region in which they were created and/or propagated. However, it may be argued that any dentin damage occurring between pre- and post-preparation conditions would be below the spatial resolution threshold of the micro-CT system, and thus may be overlooked. The full extension of dentinal microcracks visualized under conventional stereomicroscopy may also be observed in micro-CT cross-sectional images, which confirms the reliability of this novel technology for detecting dentin defects. Notably, while conventional sectioning techniques allow the evaluation of only a few slices per tooth with the possibility of missing several defects along the root, hundreds of slices of each tooth can be analyzed with 
Table III. Distribution of microcracks prior to and following preparation $(\%)$.

\begin{tabular}{lcccc}
\hline Time point & WO & OS & RE & Control \\
\hline Prior to preparation & & & & \\
Coronal part & 35 & 35 & 36 & 35 \\
Medial part & 37 & 44 & 37 & 36 \\
Apical part & 28 & 21 & 27 & 29 \\
Following preparation & & & & \\
Coronal part & 35 & 41 & 36 & 35 \\
Medial part & 37 & 36 & 37 & 36 \\
Apical part & 28 & 23 & 27 & 29 \\
\hline
\end{tabular}

WO, WaveOne; OS, OneShape; RE, Reciproc.

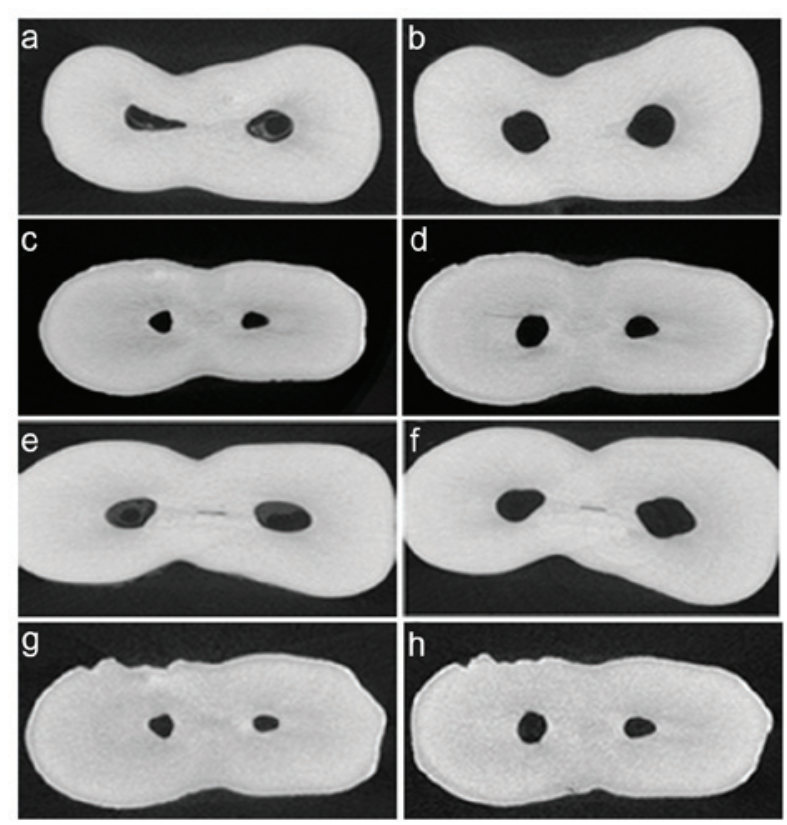

Figure 4. Micro-computed tomography images demonstrating the morphology of dentinal microcracks prior to and following preparation with different systems. (a) Prior to and (b) following preparation with the WaveOne system, microcracks were not observed. (c) Prior to preparation with the OneShape system, microcracks were observed in the dentine, and (d) microcracks increased following preparation with this system. (e) Prior to preparation with the Reciproc system, microcracks were observed in the dentine, and (f) these microcracks were not evidently increased following preparation with this system. (g) Prior to and (h) following preparation using hand files, microcracks were not observed.

micro-CT imaging $(9,28)$. Another methodologic dissimilarity between the technique used in the present study and those of previous studies is associated with sample selection. Although the majority of previous studies used single-rooted teeth, the present study used mesial canals of mandibular molars $(3,12,13,26,29)$. These canals have a constricted anatomic configuration that may result in increased stress on the dentinal surface during mechanical preparation and, consequently, increase the potential for cracks. Therefore, the current results demonstrated a marked contrast with the findings of previous studies. Comparing dentinal microcracks only subsequent to preparation demonstrated that the length increased significantly. This reflected the results identified in previous studies that did not conduct pre-preparation comparisons.

Therefore, in the present study, it is hypothesized that the influence of the prepreparation dentinal microcracks on microcrack development is significant. The condition of the prepreparation microcracks is associated with the patient's age, gender, occlusion habits and occlusion force. However, the data regarding the length of microcracks prior to and following preparation exhibited normal distribution and homogeneity of variance. Therefore, a paired t-test was used to analyze the increase in the length within the same sample and the SNK test was conducted to compare the differences between the experimental and control groups (pre- and post-preparation), respectively.

The paired t-test performed in the current system indicated that the OS system resulted in the formation of evident microcracks. By contrast, the WO system, the RE system and the hand files may not form marked microcracks. It has been reported that the potential to promote dentinal defects may be associated with the design of the instrument used (11). According to Bier et al (3), an increased file taper of rotary instruments contributed to the formation of dentinal defects due to the increased stress on the canal walls. However, the reciprocating instruments, WO and RE systems, had larger tapers in comparison with the rotary instrument, OS system. According to a recent study, reciprocating instruments would be more likely to promote the development or propagation of dentin microcracks and dentinal damage compared with rotary movement using SEM (12). This supports the argument that root canal preparation using only a single, large-tapered reciprocating instrument, which cuts substantial amounts of dentin in a short time, tends to create or aggravate the dentinal defects when compared with the conventional preparation that allows for a more progressive and slower mechanical enlargement. In the present study, it is speculated that the number of the files, the taper and the speed and torque had no effect on the formation of microcracks, whereas the preparation movement may affect the development of dentinal microcracks.

The different morphologies of dentinal microcracks, including complete and incomplete microcracks, as well as microcracks confined in the dentine (Fig. 2), are associated with the stress intensity, concentration zone and root canal wall thickness. The OS system generated microcracks in the apical and coronal parts of the root, and the most common morphology was microcracks confined in the dentine. The thread design in the medial part of the OS system is a transition region that changes from three blades to two $(24,30)$. This design may explain why no microcracks formed in the medial part. Furthermore, the apical part rapidly expands from size 10 to size 25 using a single file, which may have caused an increase in microcracks in this part.

In conclusion, the formation and development of dentinal microcracks may be associated with the movement caused by preparation, as opposed to the taper of the files. Among single-file Ni-Ti systems, WO and RE were not observed to cause evident microcracks, whereas the OS system resulted in increased microcracks. 


\section{References}

1. Arslan H, Barutcigil C, Karatas E, Topcuoglu HS, Yeter KY, Ersoy I and Ayranc1 LB: Effect of citric acid irrigation on the fracture resistance of endodontically treated roots. Eur J Dent 8: 74-78, 2014.

2. Ertas H, Sagsen B, Arslan H, Er O and Ertas ET: Effects of physical and morphological properties of roots on fracture resistance. Eur J Dent 8: 261-264, 2014.

3. Bier CA, Shemesh H, Tanomaru-Filho M, Wesselink PR and Wu M: The ability of different nickel-titanium rotary instruments to induce dentinal damage during canal preparation. J Endod 35: 236-238, 2009

4. Shemesh H, van Soest G, Wu M and Wesselink PR: Diagnosis of vertical root fractures with optical coherence tomography. J Endod 34: 739-742, 2008

5. Kishen A: Mechanisms and risk factors for fracture predilection in endodontically treated teeth. Endodont Top 13: 57-83, 2006.

6. Kubo M, Miura J, Sakata T, Nishi R and Takeshige F: Structural modifications of dentinal microcracks with human aging. Microscopy (Oxf) 62: 555-561, 2013.

7. Er K, Tasdemir T, Siso SH, Celik D and Cora S: Fracture resistance of retreated roots using different retreatment systems. Eur J Dent 5: 387-392, 2011.

8. Saber SE, Nagy MM and Schäfer E: Comparative evaluation of the shaping ability of ProTaper Next, iRaCe and Hyflex CM rotary NiTi files in severely curved root canals. Int Endod J 48 131-136, 2015

9. Pop I, Manoharan A, Zanini F, Tromba G, Patel S and Foschi F: Synchrotron light-based $\mu \mathrm{CT}$ to analyse the presence of dentinal microcracks post-rotary and reciprocating NiTi instrumentation. Clin Oral Investig 19: 11-16, 2015.

10. Sathorn C, Palamara JE and Messer HH: A comparison of the effects of two canal preparation techniques on root fracture susceptibility and fracture pattern. J Endod 31: 283-287, 2005.

11. Yoldas O, Yilmaz S, Atakan G, Kuden C and Kasan Z: Dentinal microcrack formation during root canal preparations by different NiTi rotary instruments and the self-adjusting file. J Endod 38: 232-235, 2012

12. Bürklein S, Tsotsis P and Schäfer E: Incidence of dentinal defects after root canal preparation: Reciprocating versus rotary instrumentation. J Endod 39: 501-504, 2013.

13. Liu R, Hou BX, Wesselink PR, Wu MK and Shemesh H: The incidence of root microcracks caused by 3 different single-file systems versus the ProTaper system. J Endod 39: 1054-1056, 2013.

14. Topçuoğlu HS, Düzgün S, Kesim B and Tuncay O: Incidence of apical crack initiation and propagation during the removal of root canal filling material with ProTaper and Mtwo rotary nickel-titanium retreatment instruments and hand files. J Endod 40: 1009-1012, 2014.

15. Ashwinkumar V,Krithikadatta J, Surendran S and Velmurugan $\mathrm{N}$ : Effect of reciprocating file motion on microcrack formation in root canals: An SEM study. Int Endod J 47: 622-627, 2014.

16. Arias A, Lee YH, Peters CI, Gluskin AH and Peters OA: Comparison of 2 canal preparation techniques in the induction of microcracks: A pilot study with cadaver mandibles. J Endod 40: 982-985, 2014
17. Wright HM Jr, Loushine RJ, Weller RN, Kimbrough WF, Waller J and Pashley DH: Identification of resected root-end dentinal cracks: A comparative study of transillumination and dyes. J Endod 30: 712-715, 2004.

18. Matsushita-Tokugawa M, Miura J, Iwami Y, Sakagami T, Izumi Y, Mori N, Hayashi M, Imazato S, Takeshige F and Ebisu S: Detection of dentinal microcracks using infrared thermography. J Endod 39: 88-91, 2013.

19. De-Deus G, Silva EJ, Marins J, Souza E, Neves Ade A, Gonçalves Belladonna F, Alves H, Lopes RT and Versiani MA: Lack of causal relationship between dentinal microcracks and root canal preparation with reciprocation systems. J Endod 40: 1447-1450, 2014.

20. Beling KL, Marshall JG, Morgan LA and Baumgartner JC: Evaluation of cracks associated with ultrasonic root-end preparation of gutta-percha filled canals. J Endod 23: 323-326, 1997.

21. Yared G: Canal preparation using only one Ni-Ti rotary instrument: Preliminary observations. Int Endod J 41: 339-344, 2008.

22. Pérez-Higueras JJ, Arias A and de la Macorra JC: Cyclic fatigue resistance of $\mathrm{K} 3, \mathrm{~K} 3 \mathrm{XF}$, and twisted file nickel-titanium files under continuous rotation or reciprocating motion. J Endod 39: 1585-1588, 2013.

23. Kiefner P, Ban M and De-Deus G: Is the reciprocating movement per se able to improve the cyclic fatigue resistance of instruments? Int Endod J 47: 430-436, 2014

24. Nabeshima CK, Caballero-Flores H, Cai S, Aranguren J, Borges Britto ML and Machado ME: Bacterial removal promoted by 2 single-file systems: Wave one and one shape. J Endod 40: 1995-1998, 2014

25. Bürklein S, Hinschitza K, Dammaschke T and Schäfer E: Shaping ability and cleaning effectiveness of two single-file systems in severely curved root canals of extracted teeth: Reciproc and WaveOne versus Mtwo and ProTaper. Int Endod J 45: 449-461, 2012.

26. Shemesh H, Bier C, Wu M, Tanomaru-Filho M and Wesselink PR: The effects of canal preparation and filling on the incidence of dentinal defects 42: 208-213, 2009.

27. Shemesh H, Roeleveld AC, Wesselink PR and Wu MK: Damage to root dentin during retreatment procedures. J Endod 37: 63-66, 2011.

28. Moeller L, Wenzel A, Wegge-Larsen AM, Ding M and Kirkevang LL: Quality of root fillings performed with two root filling techniques. An in vitro study using micro-CT. Acta Odontol Scand 71: 689-696, 2013.

29. Liu R, Kaiwar A, Shemesh H, Wesselink PR, Hou B and Wu M: Incidence of apical root cracks and apical dentinal detachments after canal preparation with hand and rotary files at different instrumentation lengths. J Endod 39: 129-132, 2013.

30. Kim HC, Lee MH, Yum J, Versluis A, Lee CJ and Kim BM: Potential relationship between design of nickel-titanium rotary instruments and vertical root fracture. J Endod 36: 1195-1199, 2010 . 